\title{
Intuitive understanding of the relationship between the elasticity of objects and kinematic patterns of collisions
}

\author{
Michele Vicovaro $^{1,2}$ • Luigi Burigana ${ }^{1}$
}

Published online: 9 December 2015

(C) The Psychonomic Society, Inc. 2015

\begin{abstract}
Horizontal collisions have long been used as a tool for exploring people's intuitive understanding of elementary physical laws. Here, we explored intuitive understanding of the relationship between the kinematic patterns of collisions and the elasticity of the colliding objects. In Experiment 1A, we manipulated the simulated materials of two virtually colliding spheres and asked the participants to judge whether the simulated collisions appeared "natural" or "unnatural." We did the same in Experiments 1B and 2, but asked the participants to adjust the velocities until the collisions appeared to be "perfectly natural." In Experiment 3, we removed pictorial cues to the materials of the colliding spheres and asked the participants to rate the bounciness of the materials, in view of the kinematics of simulated collisions. Overall, the results showed that observers intuitively understood that collisions between more elastic objects subtend a higher coefficient of restitution than collisions between objects with lesser elasticity. The results also highlighted some discrepancies between the intuitive and Newtonian physics of collisions. Observers were somewhat insensitive to violations of the principle of energy conservation, and their responses were influenced by irrelevant kinematic features of the collisions, such as the collision type and precollision velocity. We discuss our experimental results in relation to salient theoretical perspectives on intuitive physics.
\end{abstract}

Michele Vicovaro

michele.vicovaro@unipd.it

1 Department of General Psychology, University of Padua, Padua, Italy

2 Dipartimento di Psicologia Generale, Università di Padova, via Venezia 8, I-35131 Padova, Italy
Keywords Intuitive physics $\cdot$ Collisions $\cdot$ Causal perception · Information integration $\cdot$ Material properties

Intuitive physics concerns people's intuitive understanding of elementary laws of physics. One may expect that people's predictions about the behavior of physical objects should be quite accurate, given their abundant everyday experiences. However, research in intuitive physics reveals that this is not always the case. For instance, a significant portion of the participants in McCloskey, Caramazza, and Green's (1980) study predicted that a ball rolling inside a C-shaped tube would follow a curved path instead of a physically correct straight path when exiting from the tube. The origin of the discrepancies between intuitive and Newtonian physics constitutes an important theoretical question for perceptual and cognitive scientists. Some scholars (e.g., Proffitt \& Gilden, 1989) have maintained that the cognitive system is inherently limited, and thus "people make judgments about natural object motions on the basis of only one parameter of information that is salient in the event" (p. 384). In contrast, other scholars (e.g., Anderson, 1981, 1983) have argued that the cognitive system can integrate multiple sources of stimulus information; thus, in principle, it can deal with multidimensional mechanical events. Hecht (2001; Hecht \& Bertamini, 2000) suggested that people's interpretations of physical events are based on the "externalization" of body dynamics, rather than on the mechanical laws of motion (see also Yates et al., 1988).

A fundamental topic of Newtonian physics is the relationship between force and motion. How does an object move in response to the application of a force? People's intuitive understanding of this relation has been explored in various physical contexts, such as projectiles exiting from curvilinearshaped tubes (Cooke \& Breedin, 1994; Kaiser, Proffitt, \& Anderson, 1985; McCloskey, 1983; McCloskey et al., 1980; 
McCloskey \& Kohl, 1983). objects falling after having been released by moving carriers (Kaiser, Proffitt, \& McCloskey, 1985; Kaiser, Proffitt, Whelan, \& Hecht, 1992; Krist, 2000; McCloskey, 1983; McCloskey, Washburn, \& Felch, 1983). projectiles thrown by virtual human characters (Hecht \& Bertamini, 2000; Vicovaro, Hoyet, Burigana, \& O'Sullivan, 2014), and two-body collisions (Kaiser \& Proffitt, 1987; Michotte, 1963; Runeson, 1977/1983; White, 2007).

In this study, we explored the intuitive physics of horizontal collisions, which are physical events with the following four characteristics: (1) A collision involves two spherical bodies (which we call $A$ and $B$ ), the masses of which are uniformly distributed (e.g., billiard balls); (2) the spheres have no spin; (3) the collision takes place in a frictionless, isolated environment; and (4) the spheres move horizontally on the observer's frontal plane. Figures 1A and B depict two types of horizontal collisions.

For horizontal collisions like those represented in Figs. 1A and $\mathrm{B}$, the following equations specify the postcollision velocities of the spheres $\left(v_{A}\right.$ and $\left.v_{B}\right)$ as a function of their precollision velocities $\left(u_{A}\right.$ and $\left.u_{B}\right)$, their masses $\left(m_{A}\right.$ and $\left.m_{B}\right)$, and the so-called "coefficient of restitution" $(C)$ :

$v_{A}=\left[m_{A} u_{A}+m_{B} u_{B}+m_{B} C\left(u_{B}-u_{A}\right)\right] /\left(m_{A}+m_{B}\right)$,

$v_{B}=\left[m_{A} u_{A}+m_{B} u_{B}+m_{A} C\left(u_{A}-u_{B}\right)\right] /\left(m_{A}+m_{B}\right)$.

These equations are derived from the law of momentum conservation (see Kittel, Knight, \& Ruderman, 1973). Here we presume that a positive velocity denotes a motion from left to right, whereas a negative velocity denotes a motion in the opposite direction. The coefficient of restitution $(C)$ is a key concept for our study. The following equation is implied by the equations above and specifies $C$ as a function of the preand postcollision velocities of $A$ and $B$ :

$C=\left(v_{B}-v_{A}\right) /\left(u_{A}-u_{B}\right)$.

Newtonian mechanics implies that $0 \leq C \leq 1$. First, $C$ cannot be greater than 1 because, in that case, the kinetic energy after the collision would be greater than the kinetic energy before the collision, which would violate the principle of energy conservation. Second, $C$ cannot be smaller than 0 because this would imply the interpenetration of the colliding objects, which is clearly impossible for solid bodies. ${ }^{1}$ These bounds for $C$ imply that the relative postcollision velocity of the spheres $\left(v_{B}-v_{A}\right)$ must lie between 0 and the relative precollision velocity of the spheres $\left(u_{A}-u_{B}\right)$.

Probably due to their simplicity, horizontal collisions have long been used as a privileged case for exploring the degree of consistency between intuitive and Newtonian physics. Albert

\footnotetext{
${ }^{1}$ For example, let us presume $u_{A}>u_{B}$. If $C<0$, then $\left(v_{B}-v_{A}\right)<0$, which implies $v_{A}>v_{B}$. This would mean that object $A$ overtakes object $B$ in the collision process.
}

Michotte was a pioneer in this field of research. In his most celebrated experiment (Michotte, 1963, pp. 19-20), observers were presented with two small squares aligned horizontally (see Fig. 1A for a 3-D version of Michotte's stimuli). At a point in time, one square $(A)$ started moving toward the other $(B)$, which was initially stationary. Upon being contacted, $B$ started moving with the same velocity as $A$, whereas $A$ came to a stop. The vast majority of observers described this scene by saying that $A$ "launched" or "kicked" $B$ - that is, that the motion of $A$ had caused the motion of $B$. This phenomenon was called the launching effect. Michotte adopted a Gestalttheoretic approach to the perception of causality. He showed that the physical plausibility of stimulus collisions was neither necessary nor sufficient for perceiving the launching effect, which instead proved to depend on specific laws of perceptual organization. For instance, Michotte showed that the launching effect occurred even when the value of $C$ subtended by the simulated collision was greater than $1-$ that is, when the collision implied the violation of the laws of energy conservation. In line with Michotte's view, various researchers have highlighted inconsistencies between visual impressions of causality and the physical laws of collisions (e.g., Bae \& Flombaum, 2011; Scholl \& Nakayama, 2002; White, 2007; Yela, 1952). Furthermore, Kaiser and Proffitt (1987) showed that when simulated off-center collisions imply large deviations from the physically correct kinematic parameters, they are still judged to be "natural" by observers most of the time (see also O'Sullivan, Dingliana, Giang, \& Kaiser, 2003).

Dissenting from Michotte, some researchers have emphasized the substantial consistency between physical laws and subjective judgments of collisions. Runeson (1977/1983) argued that the visual system is "attuned" to the physics of collisions. His theoretical approach, which is referred to as the "kinematic specification of dynamics" (Runeson \& Frykholm, 1983). relies on the idea that observers can "pick up" invariant dynamic properties of colliding objects from the kinematic patterns of collisions. The consistency between subjective judgments of collisions and Newtonian mechanics was also emphasized by Twardy and Bingham (2002). in their study, the participants were presented with virtual animations depicting a ball falling from high above that, at the end of the fall, bounced (collided) several times upon the ground. The authors manipulated the value of $C$ implied by the rebounds of the ball and asked the participants to rate the "naturalness" of the ball's motion. The results showed that the naturalness ratings sharply decreased when the simulated value of $C$ was greater than 1 . This suggests that people are highly sensitive to violations of the principle of energy conservation. On the basis of these insights, Sanborn, Mansinghka, and Griffiths (2013) suggested that subjective judgments of causality are based on the assumption that the value of $C$ subtended by horizontal collisions may only vary between 0 and 1 . In a recent study, De Sá Teixeira, Oliveira, and Duarte Silva 


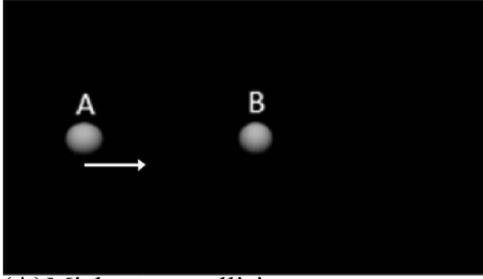

(A)Michottean collision
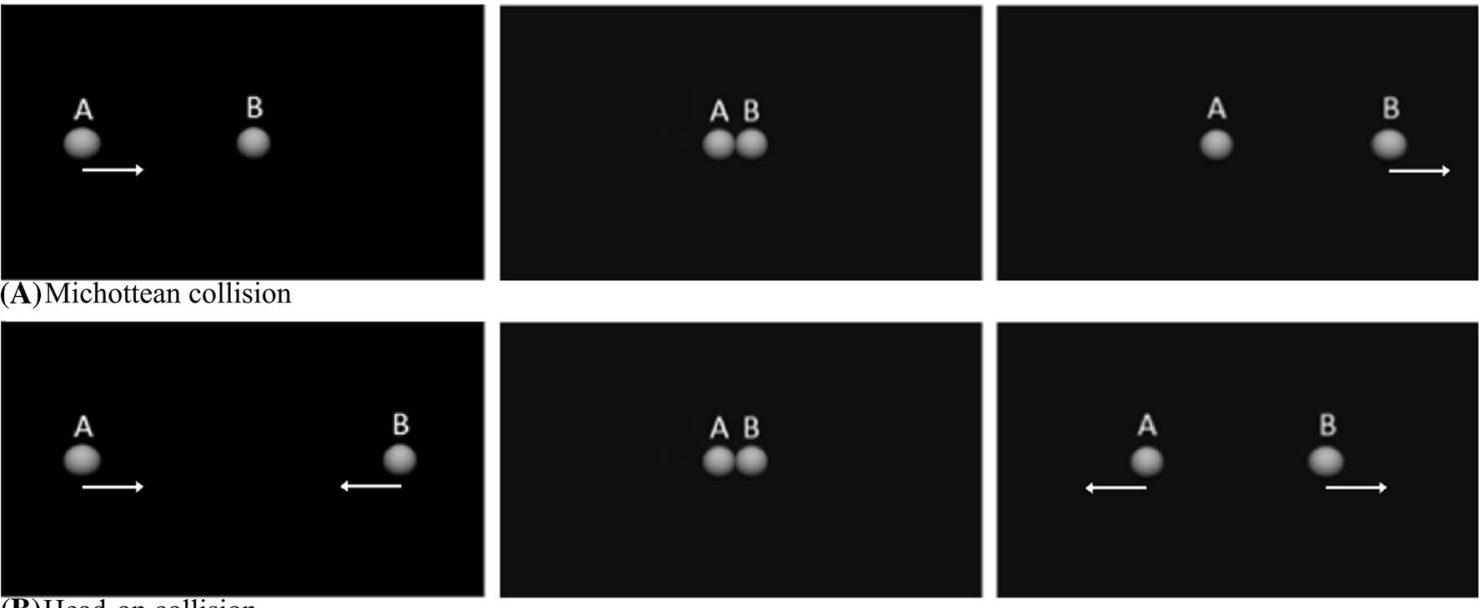

(B)Head-on collision

Fig. 1 (A) Three frames of a Michottean collision. (B) Three frames of a head-on collision. The letters $A$ and $B$ and arrows are added to indicate which objects are moving in the three stages of the collision events

(2014) found that participants' predicted outcomes of collisions in a simulated Newton's cradle device subtended values of $C$ centered around .3; they argued that this value might have been "internalized in order to reflect knowledge on the natural statistics of our ecological environment" (De Sá Teixeira et al., 2014, p. 499).

\section{From "immaterial" to "material" colliding objects}

With few exceptions, previous research on the visual perception of collisions has been conducted using "immaterial" colliding objects as experimental stimuli (e.g., simple, colorless 2-D shapes). Apart from technical reasons, this seems to be because researchers have implicitly or explicitly assumed that perceptual and cognitive judgments of collisions are not influenced by the materials from which the colliding objects are made. From a physical viewpoint, however, material properties exert a dramatic influence on objects' motion. On the one hand, the materials of two colliding objects may affect their masses, which-in turn-do influence the objects' postcollision velocities (by Eqs. 1 and 2). On the other hand, the materials of objects may also affect their elasticity values, which - in turn - do influence the postcollision velocities. For example, for horizontal collisions such as those depicted in Fig. 1, other things being equal, the relative postcollision velocity $\left(v_{B}-v_{A}\right)$ would be much smaller in a collision between Plasticine spheres (low elasticity) than in a collision between wooden spheres (high elasticity).

For collisions from common experience, the elasticity of the colliding objects has a strong influence on the resulting kinematic pattern (see Barnes, 1958, p. 8). This influence can be formalized in terms of parameter $C$ (see Eqs. 1-3). In general, for any given pair of pre-collision velocities $u_{A}$ and $u_{B}$, the smaller the elasticity of the objects, the smaller parameter
$C$, the smaller the relative postcollision velocity $\left(v_{B}-v_{A}\right)$. This was empirically demonstrated by Cross (1999). who showed that collisions involving comparatively elastic objects, such as steel balls and tennis balls, are characterized by higher values of $C$, as compared with collisions involving comparatively inelastic objects, such as Plasticine balls and baseballs. ${ }^{2}$

Our present study focused on people's intuitive understanding of the relationship between the elasticity of objects and kinematic patterns of collisions. The participants in our experiments were shown simulated collisions in which two colliding spheres had the same simulated material and the same apparent size; hence, equal implied mass. Thus, we could vary the elasticity of the colliding objects (by manipulating their simulated material) while keeping their relative mass fixed at zero $\left(m_{A}-m_{B}=0\right)$. In a recent study by Vicovaro and Burigana (2014). manipulations of the simulated materials of the colliding spheres were, instead, aimed at varying their relative masses. The results of that study showed that people have a good qualitative understanding of the relationship between the relative masses of the colliding objects and the kinematic patterns of collisions. Recently, manipulations of simulated materials have also been used for varying the implied masses of virtual objects in research on the visual perception of objects' stability (Lupo \& Barnett-Cowan, 2015).

Few studies have explored people's intuitive understandings of the relationship between the elasticity of colliding objects and the kinematic patterns of collisions. De Sá

\footnotetext{
${ }^{2}$ These examples highlight that the scientific concept of elasticity should not be confused with the concept of elasticity meant in everyday language. In everyday language, an object is only meant to be "elastic" if (besides other conditions) it is deformable - namely, it undergoes visible shape modifications consequent to the application of comparatively small forces. In contrast, the scientific concept of elasticity is independent of deformability, as is shown by the fact that physically elastic objects can either be deformable (tennis balls) or nondeformable (steel balls).
} 
Teixeira et al. (2014) asked the participants in their study to predict the outcomes of collisions occurring between pendulum spheres in a simulated Newton's cradle device. They manipulated the implied elasticity of the colliding spheres by varying their simulated materials, and found that its effect on the participants' responses was quite small. Warren, Kim, and Husney (1987) presented study participants with virtual animations depicting a ball falling from high that bounced (collided) several times upon the ground at the end of the fall. They manipulated the value of $C$ implied by the ball's rebounds and showed that the participants could precisely estimate the "bounciness" (elasticity) of the ball on the basis of the kinematics of its motion. To our knowledge, this is the only evidence so far supporting the hypothesis that people may intuitively understand the relationship between the kinematics of collisions and the elasticity of objects.

\section{Michottean and head-on collisions}

We presented the participants with two types of simulated horizontal collisions, named "Michottean" and "head-on." The former was extensively used by Michotte (1963) in his seminal work on the visual perception of causality; it is characterized by the fact that sphere $B$ is stationary during the precollision phase $\left(u_{B}=0\right.$; see Fig. 1A). If $m_{A}=m_{B}$, then for Michottean collisions, Eqs. 1 and 2 simplify as follows:

$v_{A}=u_{A}(1-C) / 2$,
$v_{B}=u_{A}(1+C) / 2$.

These equations show that, after the collision, both spheres move in the same direction, which is the same as that of $A$ before the collision. The equations also show that $v_{A}$ decreases with $C$ and may vary between 0 (when $C=1$ ) and $.5 u_{A}$ (when $C=0$ ), whereas $v_{B}$ increases with $C$ and may vary between $.5 u_{A}$ (when $\left.C=0\right)$ and $u_{A}$ (when $\left.C=1\right)$. For instance, if $C=0$, after the collision both spheres move with the same velocity, which is $.5 u_{A}$. If instead $C=1$, sphere $A$ remains stationary after the collision, whereas sphere $B$ moves with the same velocity that $A$ had before the collision.

Head-on collisions are characterized by the fact that the spheres move toward each other with equal absolute velocities ( $u_{A}=-u_{B}$; see Fig. 1B) during the precollision phase. If $m_{A}=m_{B}$, for head-on collisions, Eqs. 1 and 2 come down to

$v_{A}=-u_{A} C$,

$v_{B}=u_{A} C$.

These equations mean that, after the collision, both spheres move in opposite directions with equal absolute velocities, which increases as $C$ increases (in particular, $v_{A}=v_{B}=0$ when $C=0$, and $v_{A}=-v_{B}=-u_{A}$ when $C=1$ ).

\section{Experiment 1A}

In Experiment 1A, we presented the participants with virtually simulated Michottean and head-on collisions and manipulated the simulated materials of the colliding spheres, which could be wood, polystyrene, or Plasticine. The size of the spheres was kept constant throughout the experiment, and the two spheres involved in one collision always had the same simulated material. This implies that spheres $A$ and $B$ had the same implied mass in each simulated collision, allowing us to apply Eqs. 4-7, which presume $m_{A}=m_{B}$. We created six experimental conditions, resulting from a 2 Collision Type (Michottean, head-on) $\times 3$ Simulated Material of the Spheres (wood, polystyrene, Plasticine) factorial design. Across the six experimental conditions, the precollision velocities of the spheres $\left(u_{A}\right.$ and $u_{B}$ ) were kept constant, and the $C$ coefficient implicit in each collision was systematically varied within the interval from 0 to 2 by varying the postcollision velocities $v_{A}$ and $v_{B}$ (see Eqs. 4-7). Thus, both physically plausible $(0 \leq C \leq 1)$ and implausible $(C>1)$ simulated collisions were presented to the participants. The participants were asked to judge whether each simulated collision appeared "natural" or "unnatural."

Using the method described in the Experimental Design section, we determined the most natural coefficient of restitution $(N C)$ - defined as the implicit value of $C$ in collisions with the greatest probability of appearing "natural" - for each participant and experimental condition. By the method described in the Appendix, we also determined the physical coefficient of restitution (PC) - defined as the implied coefficient of restitution by collisions between physical objects of real wood, polystyrene, or Plasticine-for each of the six experimental conditions. We found that the $P C$ was independent of the collision type (i.e., it was the same for Michottean and head-on collisions) and varied with the spheres' material. The values of $P C$ were .66 for collisions between wood spheres, .61 for collisions between polystyrene spheres, and .13 for collisions between Plasticine spheres. These measures show that wood and polystyrene spheres have similar elasticity and are both more elastic than Plasticine spheres.

The comparison between the subjective measure $N C$ and the physical measure $P C$ is a key step in testing the experiment's main hypothesis - that is, that people have a good intuitive understanding of the relationship between the object's elasticity and the kinematic patterns of the collisions. If this were true, then the $N C$ should be greater when the simulated material of the spheres is wood $(P C=.66)$ or polystyrene $(P C=.61)$ than when it is Plasticine $(P C=.13)$, and should be independent of the collision type (Michottean, head-on). We note two reasons why wood, polystyrene, and Plasticine are suitable materials for testing this hypothesis. One reason is that they are common in everyday life, and thus it is likely that the participants may have good intuitive knowledge of their material properties. The other reason is that they allowed us to 
test whether the participants' naturalness judgments were "correctly" based on the material properties of the colliding spheres (e.g., elasticity), rather than on their masses. ${ }^{3}$

Another hypothesis to be tested by our experiment related to the participants' sensitivity to violations of the principle of energy conservation. If the participants were sensitive to such violations (as was argued by Twardy \& Bingham, 2002). then simulated collisions subtending values of $C$ greater than 1 should appear decidedly "unnatural."

\section{Method}

Participants Twenty psychology students at the University of Padua (ages 20-38 years; 13 females, seven males) participated in the experiment on a voluntary basis. They all had normal or corrected-to-normal visual abilities. On average, they had studied physics at school for 2.8 years $(S D=1.64$ years $)$.

Stimuli and apparatus The stimuli were presented on a personal computer equipped with a $37.5 \times 30 \mathrm{~cm}$ CRT screen and a keyboard. The participants sat at a distance of about $50 \mathrm{~cm}$ from the screen, the background of which was black. Two simulated spheres of equal size were presented at the middle height of the screen, with their centers aligned horizontally. Their apparent size, computed from the diameters of their corresponding images on the screen $(2.52 \mathrm{~cm})$, was $8.4 \mathrm{~cm}^{3}$, subtending a visual angle of about $2.88^{\circ}$. For the Michottean collisions (see Fig. 1A), at the beginning of each animation, one sphere $(A)$ appeared close to the left edge of the screen and the other sphere $(B)$ appeared in the center. Then, $170 \mathrm{~ms}$ after the appearance of the spheres, $A$ began to move horizontally from left to right toward $B$, uniformly and without rotation, until it made contact with $B$. The postcollision velocities of the spheres in a trial were computed using Eqs. 4 and 5, after inserting into them the value of $C$ specified (by the experimental plan) for that trial. For head-on collisions (see Fig. 1B), at the beginning of each animation, one sphere $(A)$ appeared close to the left edge of the screen and the other sphere $(B)$ appeared close to the right edge. Then, $170 \mathrm{~ms}$ after their appearance, both spheres started moving simultaneously with opposite velocities until they made contact with each other in the center of the screen. The postcollision velocities were computed using Eqs. 6 and 7. Each animation lasted 2, $150 \mathrm{~ms}$ (the postcollision phase lasted $1,000 \mathrm{~ms}$ ). The precollision velocities of the spheres were kept the same throughout the experiment $\left(A=8.6 \mathrm{~cm} \mathrm{~s}^{-1}, B=0 \mathrm{~cm} \mathrm{~s}^{-1}\right.$ for

\footnotetext{
${ }^{3}$ We argue, in this regard, that although masses are generally salient in perception, they should not affect the kinematic patterns of collisions if $m_{A}=m_{B}$. For instance, the kinematic pattern of a horizontal collision involving two wood spheres is similar to that of a horizontal collision involving two polystyrene spheres $(P C=.66$ and .61 , respectively), even though the former are rather different in mass from the latter.
}

Michottean collisions; $A=8.6 \mathrm{~cm} \mathrm{~s}^{-1}, B=-8.6 \mathrm{~cm} \mathrm{~s}^{-1}$ for head-on collisions).

We jointly manipulated the simulated materials of the spheres involved in the same simulated collision; these could be wood, polystyrene, or Plasticine. The spheres were created with 3D Studio Max. Photographic textures depicting the simulated materials were attached to the spheres' surfaces, and their reflectances were regulated in order to increase the similarity with their corresponding real spheres (see the next paragraph). The spheres thus created are depicted in Fig. 2. In each of the six experimental conditions ( 2 Collision Types $\times 3$ Simulated Materials), we varied the value of $C$ underlying the collisions, as was required by the method of "randomly interleaved staircases" described in the Experimental Design section below.

The participants were allowed to touch and grasp real spheres made of wood, polystyrene, and Plasticine before and during the experiment. The masses of these spheres were 55,5 , and $80 \mathrm{~g}$, respectively, and their diameters were $4.5 \mathrm{~cm}$. The real Plasticine sphere had a bright orange color similar to that of the corresponding simulated sphere (see Fig. 2). These real spheres were meant to facilitate the identification of the corresponding simulated materials of the virtual spheres and were also used to compute the $P C$ for each of the six experimental conditions (see the Appendix).

Procedure Prior to this and the following experiments, the participants read and signed informed consent forms that had been approved by the local ethics committee (Department of General Psychology, University of Padua). The instructions on the screen informed the participants that they would be presented with two simulated colliding spheres, which could be made of any one of three materials: wood, polystyrene, or Plasticine. At that point, the participants were allowed to touch the real spheres set besides the keyboard and were told that the spheres shown on the screen would faithfully represent those real spheres. The participants were asked to judge whether each presented collision was "natural" or "unnatural." The instructions specified that a collision had to be judged as "natural" or "unnatural" depending on its physical plausibility (or implausibility). The instructions further specified that a collision had to be judged "unnatural" when the postcollision motion of one or both spheres appeared to be too fast or too slow, compared with their precollision motion. In each trial, the participants were allowed to view the stimulus as many times as they wanted by pressing the spacebar on the keyboard; when they felt ready to respond, they had to press "N" for the "natural" response or " $\mathrm{Z}$ " for the "unnatural" one. After reading the instructions, the participants were presented with five randomly chosen stimuli to familiarize them with the task.

Experimental design We determined an upper naturalness bound and a lower naturalness bound for each participant and 

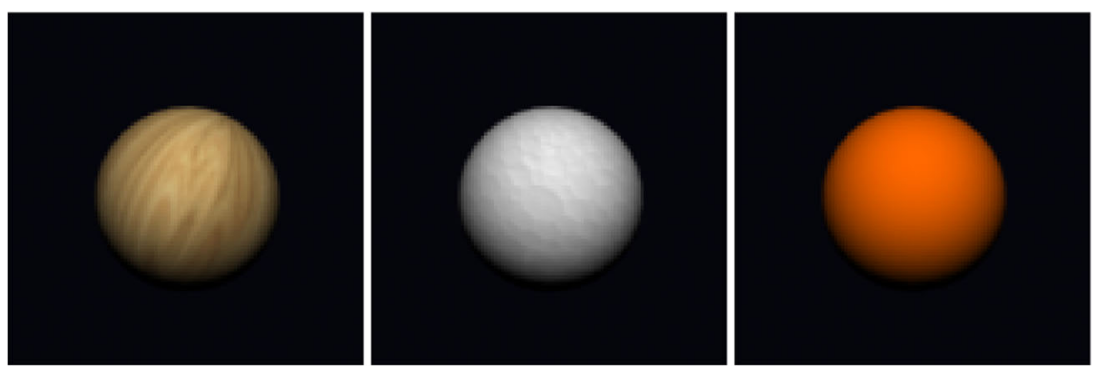

Fig. 2 Simulated spheres used as stimuli in Experiments 1A and 1B. The simulated materials are, from left to right, wood, polystyrene, and Plasticine. To view this figure in color, please see the online issue of the journal

each experimental condition. The upper naturalness bound may be interpreted as the value of $C$ above which the collision appeared "unnatural" to the participant more than $50 \%$ of the time, presumably because the postcollision velocities of one or both spheres looked too fast relative to their precollision velocities. Symmetrically, the lower naturalness bound may be interpreted as the value of $C$ below which the collision appeared "unnatural" to the participant more than $50 \%$ of the time, presumably because the postcollision velocities of one or both spheres looked too slow relative to their precollision velocities. The naturalness interval was the interval of values of $C$ that lay between the two bounds and gave rise to the impression of a "natural" collision more than $50 \%$ of the time. Finally, we defined the most natural coefficient of restitution $(N C)$ as the midpoint of the naturalness interval. Assuming that the "naturalness" judgments were normally distributed, $N C$ was tantamount to the value of $C$ implicit in a collision with the greatest probability of appearing "natural."

We used the method of "randomly interleaved staircases" to estimate the individual upper and lower naturalness bounds (Levitt, 1971). In each of the six experimental conditions, we varied the implicit value of $C$ in the collision, which could take on 21 possible values, ranging from 0 to 2 in steps of 0.1 . Figure 3 depicts an example of the randomly interleaved staircases procedure. The precollision velocities of the spheres were fixed (see the Stimuli and Apparatus section above), whereas their postcollision velocities were varied as a function of $C$, by applying Eqs. 4 and 5 for Michottean collisions, and Eqs. 6 and 7 for head-on collisions.

Both naturalness bounds were estimated by generating two staircases. For estimating the individual upper naturalness bounds, one staircase started from $C=1$. Every time the participant responded by judging the collision "natural," $C$ was increased by one step; every time the participant responded by judging the collision "unnatural," $C$ was decreased by one step. Thus, the staircase changed its direction whenever the participant changed his or her answer, and it continued in that direction until the participant changed his or her answer again. Symmetrically, the other staircase started from $C=2$. The value of $C$ was decreased as long as the participant chose the "unnatural" response, and the staircase changed its direction whenever the participant changed his or her response. A "run" in a staircase was a sequence of steps between two consecutive changes in direction. Both staircases were terminated after eight runs. ${ }^{4}$ The individual upper naturalness bounds were estimated by averaging the values of $C$ at the midpoints of the last four runs of both staircases (Levitt, 1971, p. 470).

We applied the same procedures to estimate the individual lower naturalness bounds, with one staircase starting from $C=0$ and the other from $C=1$. Each staircase was increased by one step after an "unnatural" response and decreased by one step after a "natural" response. Both staircases were terminated after eight runs, and the final (averaging) computations were the same as those described above.

Individual upper and lower naturalness bounds were estimated in each of the six ( 2 Collision Types $\times 3$ Simulated Materials) experimental conditions. The experiment was divided into two blocks: Half of the participants received the 12 staircases (3 Simulated Materials $\times 2$ Bounds $\times 2$ Staircases) relative to Michottean collisions first, whereas the other half received the 12 staircases relative to head-on collisions first. The 12 staircases in each block were randomly interleaved to avoid anticipatory effects. The participants were allowed to rest as much as they wanted after the first block. The experimental session could last from 25 to $35 \mathrm{~min}$.

\section{Results and discussion}

Figure 4 shows the naturalness intervals (averaged across participants) obtained in the six conditions of the experiment. We marked four points for each interval: the mean upper naturalness bound (top of the interval), the mean lower naturalness bound (bottom of the interval), the most natural coefficient of restitution (NC; midpoint of the interval, thick line), and the physical coefficient of restitution $(P C$, dashed line). The location of the naturalness intervals along the $C$ continuum was higher when the simulated material of the colliding spheres was wood or polystyrene than when it was Plasticine. Moreover, the location of the naturalness intervals on the $C$ continuum was higher for Michottean collisions than for head-

\footnotetext{
${ }^{4}$ Because of the adaptive nature of the psychophysical method used in Experiment 1A, the number of trials for each participant and each staircase varied.
} 


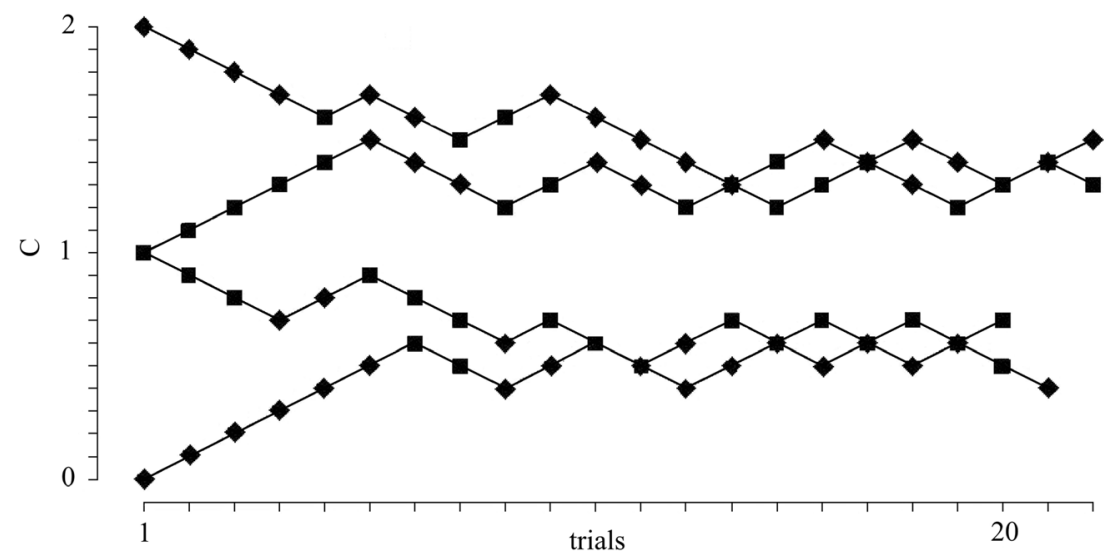

Fig. 3 Full record of the responses of a hypothetical participant tested with randomly interleaved staircases. On the horizontal axis are the trials; on the vertical axis are the 21 possible values of $C$. The small squares and diamonds represent "natural" and "unnatural" responses, respectively

on collisions, but this difference decreased in passing from wood to polystyrene to Plasticine.

We performed a two-way within-participants analysis of variance (ANOVA; with the factors Collision Type and Simulated Material) on the $N C$ measures to test this evidence statistically. The main effects of both factors were significant: $F(1,19)=14.69, p<.01, \eta_{\mathrm{p}}{ }^{2}=.44$, and $F(2,38)=19.95$, $p<.001, \eta_{\mathrm{p}}{ }^{2}=.51$, respectively. Their interaction effects were marginally significant, $F(2,38)=3.06, p=.058, \eta_{\mathrm{p}}{ }^{2}=.14$. Bonferroni post-hoc comparisons (see Table 1) showed that, for both Michottean and head-on collisions, there was no significant difference between the $N C$ s for wood and polystyrene, which were both significantly greater than the $N C$ for Plasticine. These comparisons also showed that when the simulated material was wood, but not when it was polystyrene or Plasticine, the $N C$ for the Michottean collision was larger than the $N C$ for the head-on collision.

These results reveal that the participants intuitively understood that collisions between somewhat elastic objects such as wood or polystyrene spheres imply higher values of $C$ compared with collisions between less elastic objects such as
Plasticine spheres. This finding supports the hypothesis that people intuitively understand that the kinematic patterns of collisions are prominently influenced by the elasticity of the colliding objects. Furthermore, the similarity between wood and polystyrene in the $N C$ measure and their $N C$ s over that of Plasticine support the idea that the participants correctly based their naturalness judgments on the material properties of the spheres (e.g., elasticity), rather than on their apparent masses.

Figure 4 also shows that the naturalness intervals of all six experimental conditions include values of $C$ much higher than the $P C$ s. The values of the $N C$ s averaged over the participants were $0.15-0.4$ points higher than the corresponding $P C$ values. In the case of Michottean collisions between wood or polystyrene spheres, the collisions implying values of $C$ much larger than 1 were judged to be "natural" by the participants most of the time, even though such collisions were at odds with the principle of energy conservation. This finding is inconsistent with the claim that people are highly sensitive to violations of energy conservation laws (Twardy \& Bingham, 2002). as well as with the view that people may have "internalized" a physically plausible value of $C$ "in order to reflect knowledge on the

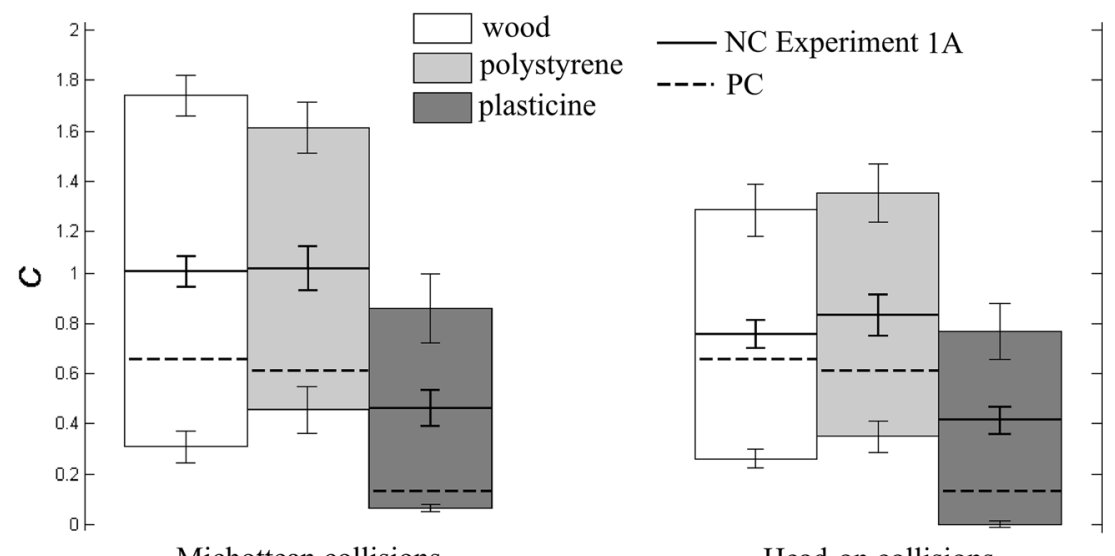

Michottean collisions

Head-on collisions

Fig. 4 Mean naturalness intervals, mean NCs, and PCs for the six combinations of the Collision Type and Simulated Material of the Spheres factors from Experiment $1 \mathrm{~A}$. The vertical bars represent the standard errors of the means 
Table 1 Results of the Bonferroni-corrected post-hoc paired comparisons for the six experimental conditions of Experiment 1A

\begin{tabular}{llllll}
\hline & (H, Wood) & (M, Pol.) & (H, Pol.) & (M, Pla.) & (H, Pla.) \\
\hline (M, Wood) & $\boldsymbol{t}=\mathbf{3 . 9 5 5}$ & $t=0.108$ & $t=1.764$ & $\boldsymbol{t}=\mathbf{7 . 2 8 9}$ & $\boldsymbol{t}=\mathbf{8 . 0 3 2}$ \\
& $\boldsymbol{p}<. \mathbf{0 0 1}$ & $p=.915$ & $p=.094$ & $\boldsymbol{p}<. \mathbf{0 0 1}$ & $\boldsymbol{p}<. \mathbf{0 0 1}$ \\
(H, Wood) & & $t=-2.510$ & $t=-0.807$ & $\boldsymbol{t}=\mathbf{3 . 7 7 0}$ & $\boldsymbol{t}=\mathbf{5 . 3 7 0}$ \\
& & $p=.021$ & $p=.43$ & $\boldsymbol{p}=\mathbf{. 0 0 1}$ & $\boldsymbol{p}<\mathbf{. 0 0 1}$ \\
(M, Pol.) & & & $t=2.333$ & $\boldsymbol{t}=\mathbf{4 . 3 4 3}$ & $\boldsymbol{t}=\mathbf{5 . 4 9 2}$ \\
& & & $p=.031$ & $\boldsymbol{p}<. \mathbf{0 0 1}$ & $\boldsymbol{p}<\mathbf{. 0 0 1}$ \\
(H, Pol.) & & & & $t=-3.173$ & $\boldsymbol{t}=\mathbf{4 . 2 5 7}$ \\
& & & & $p=.005$ & $\boldsymbol{p}<\mathbf{. 0 0 1}$ \\
(M, Pla.) & & & & & $t=1.043$ \\
& & & & & $p=.31$ \\
\hline
\end{tabular}

$M$ and $H$ stand for Michottean and head-on collisions, and Pol. and Pla. stand for polystyrene and Plasticine, respectively. For instance, (M, Pol.) stands for Michottean collisions with polystyrene spheres. Each $t$ test had 19 degrees of freedom, and the corrected value of $\alpha$ was .0033 , which resulted from dividing the original value of $\alpha(.05)$ by the number of paired comparisons (15). In this and the following tables, bold type stands for statistically significant differences

natural statistics of our ecological environment" (De Sá Teixeira et al., 2014, p. 499). Figure 4 also shows that, at variance with predictions from physics, the average $N C$ s tended to be higher for Michottean than for head-on collisions; such differences in $N C$ reached statistical significance only for collisions between wood spheres (see Table 1). Overall, the results of our experiment suggest that people intuitively understand that objects' materials affect the kinematic patterns of collisions, but people's representations of the collision behavior of objects are not fully consistent with physics.

\section{Experiment 1B}

In Experiment 1A, we used an "indirect" method to obtain the $N C$ measures: We first determined individual upper and lower naturalness bounds by using randomly interleaved staircases, and then computed the $N C$ as the arithmetic mean of both bounds. In Experiment 1B, we obtained the $N C$ using a "direct" method - namely, a variant of the psychophysical method of adjustment (Kingdom \& Prins, 2010, pp. 48-51). In six experimental conditions, which were the same as those in Experiment $1 \mathrm{~A}$, the participants were asked to adjust the value of $C$ (by adjusting the postcollision velocities) until a simulated collision appeared to be "perfectly natural." Experiment $1 \mathrm{~B}$ allowed us to test whether the results of Experiment 1A would be consistent across different psychophysical methods.

\section{Method}

Participants Twenty psychology students at the University of Padua (ages 20-36 years; 13 female, seven males) participated in the experiment on a voluntary basis. They all had normal or corrected-to-normal visual abilities. None of them had participated in Experiment 1A. On average, they had studied physics at school for 3.25 years $(S D=1.48$ years $)$.

Stimuli and apparatus The stimuli and apparatus were the same as those of Experiment 1A.

Procedure The general instructions were the same as those in the previous experiment. In addition, the participants were told that the difference between the postcollision velocities of the spheres would sometimes appear to be too large or too small as compared to the difference between their precollision velocities, and that their task was to change the difference between the postcollision velocities until the collision appeared to be perfectly "natural" - that is, as similar as possible to how a real physical collision should be. The participants were told that the difference between the postcollision velocities could be increased or decreased by pressing " $\mathrm{M}$ " or " $\mathrm{Z}$ " on the keyboard, respectively, and that they had to press ENTER when the collision appeared to be perfectly "natural." In each trial, the participants were allowed to view the stimulus as many times as they wanted by pressing the spacebar on the keyboard.

Experimental design The parameter $C$ implicit in the simulated collisions could take on 21 different values, ranging from 0 to 2 in steps of 0.1 . In each of the six experimental conditions, individual $N C$ s were obtained by generating four "series" of trials. Two series started from $C=2$, and the other two started from $C=0$. In each series, whenever the participant responded by pressing " $\mathrm{M}$ " on the keyboard, indicating that the difference between the spheres' postcollision velocities had to be increased, $C$ was increased by one step (which, by Eqs. 4 and 5, or 6 and 7, implied increasing that difference). Conversely, $C$ was decreased by one step whenever the participant responded by pressing " $\mathrm{Z}$," indicating that the difference between the postcollision velocities had to be decreased. Each series was terminated when the participant pressed ENTER, indicating that the collision appeared perfectly "natural." Individual NCs were estimated by averaging the last values of $C$ in the four series (see note 4 ).

The experiment was divided into two blocks: Half of the participants received the 12 series (3 Simulated Materials $\times 4$ Series) relative to Michottean collisions first, and the other half received the 12 series relative to head-on collisions first. The 12 series in each block were presented in random order. The participants were allowed to rest as much as they wanted after the first block. The experimental session could last from 20 to $30 \mathrm{~min}$.

\section{Results and discussion}

Figure 5 shows the $N C$ measures (averaged across participants) obtained from each of the experiment's six conditions. 


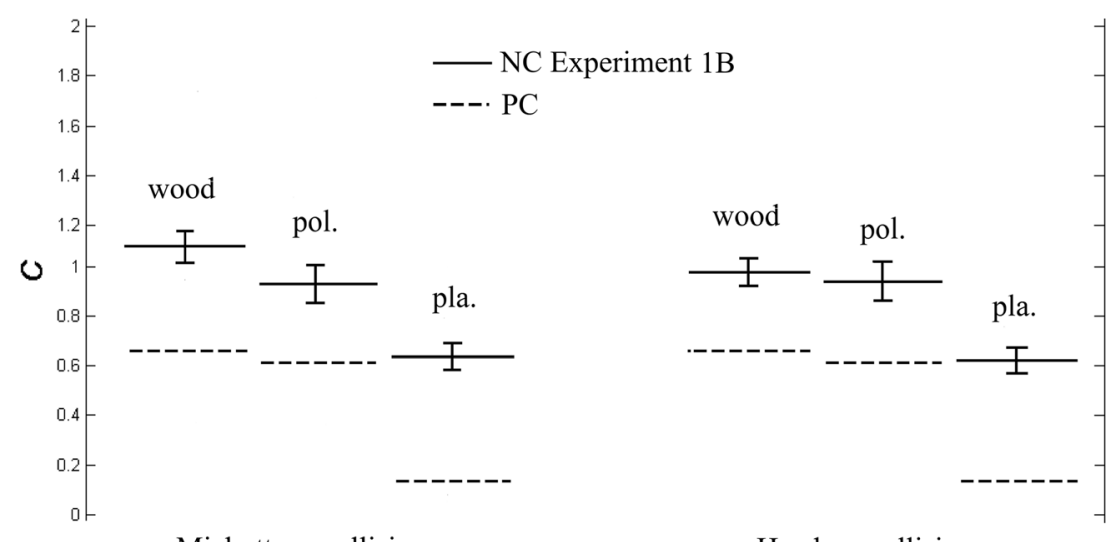

Michottean collisions

Head-on collisions

Fig. 5 Mean $N C$ s and $P C$ s for the six combinations of the Collision Type and Simulated Material of the Spheres factors from Experiment 1B. The vertical bars represent the standard errors of the means

The locations of $N C$ s along the $C$ continuum are similar to those obtained from Experiment 1A, since they were higher when the simulated material of the colliding spheres was wood or polystyrene than when the material was Plasticine. Moreover, the $N C$ is greater than the corresponding $P C$ in each of the six experimental conditions. Unlike the results from Experiment 1A, the locations of the NCs do not vary with collision type.

We performed a two-way within-participants ANOVA (with the factors Collision Type and Simulated Material) on the individual $N C$ measures. The main effects of the Collision Type factor were not significant, $F(1,19)=0.80, p>.1$, $\eta_{\mathrm{p}}{ }^{2}=.04$, whereas the main effects of the Simulated Material factor were significant, $F(2,38)=26.52, p<.001$, $\eta_{\mathrm{p}}{ }^{2}=.58$. Their interaction effects were not significant, $F(2,38)=1.90, p>.1, \eta_{\mathrm{p}}{ }^{2}=.09$. Bonferroni post-hoc comparisons (see Table 2) showed no significant difference between the $N C$ s for wood and polystyrene, which were both significantly greater than the $N C$ for Plasticine.

Overall, the results of Experiment 1B confirmed the main outcomes of Experiment 1A. The participants showed a good qualitative understanding that collisions between somewhat elastic objects (such as wood or polystyrene spheres) imply higher values of $C$ than collisions between less elastic objects (such as Plasticine spheres). Moreover, the values of the $N C$ s

Table 2 Results of the Bonferroni-corrected post-hoc paired comparisons for the three levels of the Simulated Material factor in Experiment 1B

\begin{tabular}{lll}
\hline & Polystyrene & Plasticine \\
\hline Wood & $t=1.863 p=.07$ & $\boldsymbol{t}=\mathbf{1 1 . 1 3 7}$ \\
& & $\boldsymbol{p}<.001$ \\
Polystyrene & $\boldsymbol{t}=\mathbf{5 . 9 4 4}$ \\
& & $\boldsymbol{p}<\mathbf{. 0 0 1}$ \\
\hline
\end{tabular}

Each $t$ test had 39 degrees of freedom, and the corrected value of $\alpha$ was .0167 (i.e., $.05 / 3$ ) averaged across participants were 0.3 to 0.5 points higher than the corresponding values of the $P C \mathrm{~s}$. Thus, the main features of our experimental results proved consistent across different psychophysical methods.

\section{Experiment 2}

The $P C$ measures that we obtained by the method described in the Appendix indicated that the collision type (Michottean, head-on) did not affect the value of $C$ implicit in physical collisions. By contrast, the results of Experiment 1A showed that the $N C$ measures tended to be higher for Michottean than for head-on collisions. This result was not replicated in Experiment 1B. Barnes (1958, pp. 7-8) pointed out that, besides the collision type, the objects' precollision velocities are also physically irrelevant to the value of $C$, at least when the velocity varies within a relatively narrow range of values (on the order of $0-10 \mathrm{~cm} \mathrm{~s}^{-1}$ ). The possible effects of this variable on the $N C$ measures were not tested in Experiments 1A and $1 \mathrm{~B}$, because the precollision velocity was kept constant throughout both experiments. In Experiment 2, we obtained the $N C$ measures by a psychophysical method similar to that used in Experiment 1B, but we varied both the collision type (Michottean, head-on) and the precollision velocity of the colliding objects (fast, slow) according to a factorial design. If the participants' judgments of the naturalness of collisions were consistent with the Newtonian rules of collisions, then the $N C$ measures should not vary with the collision type or the precollision velocity, because these variables are irrelevant to the value of $C$ implicit in collisions.

In Experiment 2, we also used a larger sample of simulated materials for the colliding objects than had been used in Experiments 1A and 1B. This approach was meant to test the robustness and generalizability of the main outcomes of those experiments (i.e., a consistent association between the elasticity of objects and the kinematic patterns of collisions). 
The simulated colliding objects could be super balls $(P C=$ .94), table tennis balls $(P C=.80)$, tennis balls $(P C=.71)$, rubber balls (from a beach rackets set, $P C=.37$ ), terracotta spheres $(P C=.28)$, and Plasticine spheres $(P C=.13)$. These objects were chosen because they are somewhat common in everyday life and because their $P C$ measures are quite uniformly distributed over the range of possible values of the parameter $C$.

We created 24 experimental conditions, resulting from a 2 Collision Type (Michottean, head-on) $\times 2$ Precollision Velocity (fast, slow) $\times 6$ Simulated Colliding Object (super balls, table tennis balls, tennis balls, rubber balls, terracotta spheres, Plasticine spheres) factorial design. The two spheres involved in one collision always had the same simulated material and size. In each of the 24 experimental conditions, the $N C$ measure was obtained by a method similar to that used in Experiment 1B (i.e., a variant of the adjustment method).

\section{Method}

Participants Twenty psychology students at the University of Padua (ages 19-29 years; 15 females, five males) participated in the experiment on a voluntary basis. They all had normal or corrected-to-normal visual abilities, and none of them had participated in Experiment 1A or 1B. On average, they had studied physics at school for 2.9 years $(S D=1.32$ years $)$.

Stimuli and apparatus The stimuli and apparatus were the same as in Experiments 1A and 1B, except for the following aspects. The simulated colliding objects varied not only in photographic texture and reflectance, but also in size. Simulated tennis balls had the largest size $\left(11.74 \mathrm{~cm}^{3}\right)$, since their diameter measured on the screen was $2.82 \mathrm{~cm}$, subtending a visual angle of $3.23^{\circ}$. The size of the super balls, terracotta spheres, and Plasticine spheres was $8.4 \mathrm{~cm}^{3}$ (diameter $=2.52 \mathrm{~cm}$, visual angle $=2.88^{\circ}$ ). Finally, the size of the table tennis and rubber balls was $4.34 \mathrm{~cm}^{3}$ (diameter $=$ $2.22 \mathrm{~cm}$, visual angle $=2.54^{\circ}$ ). The spheres thus created are depicted in Fig. 6. The size, photographic texture, and reflectance of the simulated objects were meant to increase the realism of their appearance and the similarity with their corresponding real objects (see the next paragraph). The precollision velocity of sphere $A$ could be either 6.7 or $11.9 \mathrm{~cm} \mathrm{~s}^{-1}$ in both the Michottean and head-on collisions. The precollision velocity of $B$ was null in the Michottean collisions, whereas it could be either -6.7 or $-11.9 \mathrm{~cm} \mathrm{~s}^{-1}$ in the head-on collisions.

As in Experiments $1 \mathrm{~A}$ and $1 \mathrm{~B}$, the real objects were meant to facilitate the identification of the corresponding simulated colliding objects, and they were also used to compute the $P C$ for each of the 24 experimental conditions (see the Appendix). The diameter of the real tennis balls was $6 \mathrm{~cm}($ mass $=58 \mathrm{~g})$; that of the super balls, terracotta spheres, and Plasticine spheres was $4.5 \mathrm{~cm}$ (masses $=40$, 105 , and $80 \mathrm{~g}$, respectively); and the diameter of table tennis and rubber balls was $3.5 \mathrm{~cm}$ (masses $=2$ and $12 \mathrm{~g}$, respectively).

Procedure The procedure was the same as that of Experiment $1 \mathrm{~B}$ (i.e., a variant of the method of adjustment).

Experimental design The experimental design was the same as that of Experiment 1B, except that in each of the 24 experimental conditions, individual $N C$ s were obtained by generating two "series" of trials rather than four. One series started from $C=2$, and the other started from $C=0$. With respect to Experiment 1B, we decreased the number of "series" in order to maintain the experiment at a reasonable length, given the increased number of experimental conditions. The experiment was divided into two blocks: Half of the participants received the 24 series $(2$ Series $\times 2$ Precollision Velocities $\times 6$ Simulated Colliding Objects) for Michottean collisions first, and the other half received the 24 series for head-on collisions first. The 24 series in each block were presented in random order. The participants were allowed to rest as much as they wanted after the first block. The experimental session could last from 30 to $40 \mathrm{~min}$.

\section{Results and discussion}

Figure 7 shows the $N C$ measures (averaged across participants) as a function of the $P C$ measures for the six levels of Simulated Colliding Objects factor (horizontal axis) and each combination of levels of the Collision Type and Precollision Velocity factors (separate curves). Overall, the locations of the $N C$ s along the $C$ continuum increased with the $P C$ s of the six
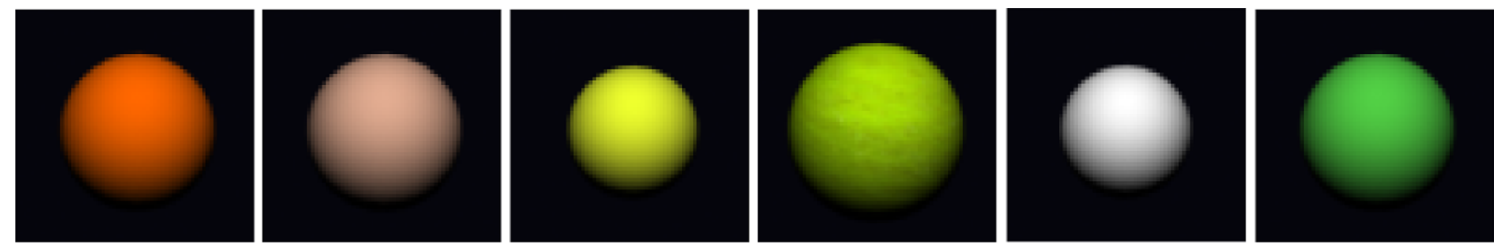

Fig. 6 Simulated objects used as stimuli in Experiment 2. The simulated objects are, from left to right, a Plasticine sphere, terracotta sphere, rubber ball, tennis ball, table tennis ball, and super ball. To view this figure in color, please see the online issue of the journal 


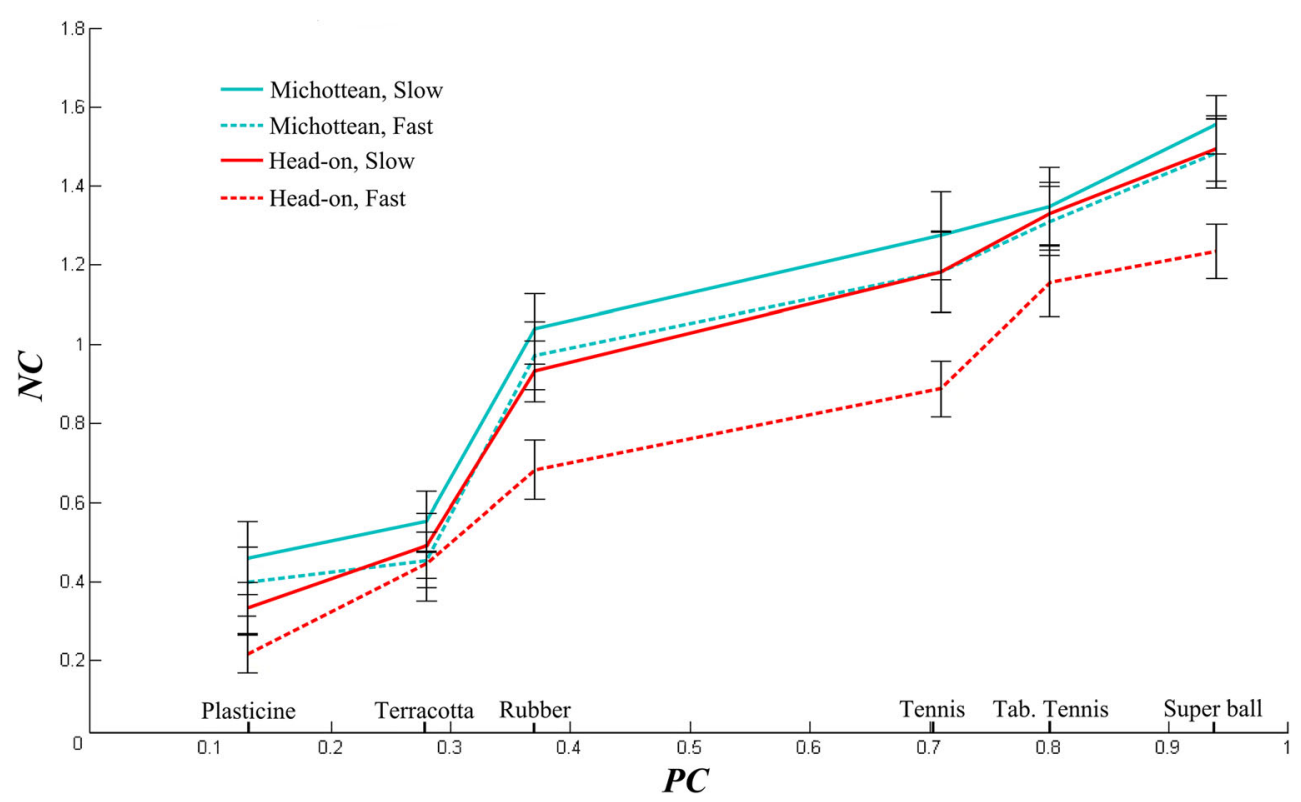

Fig. 7 Mean $N C$ measures from Experiment 2, represented as a function of the $P C$ of each type of simulated colliding object, for each combination of the Collision Type and Precollision Velocity factors. The vertical bars represent the standard errors of the means

types of simulated colliding objects. ${ }^{5}$ Moreover, the vertical separation of the curves revealed that the location of the $N C \mathrm{~s}$ was also conditional on Collision Type and Precollision Velocity. Specifically, the $N C$ s tended to increase in passing from head-on to Michottean collisions and from fast to slow in precollision velocity.

We performed a three-way within-participants ANOVA (with the Collision Type, Precollision Velocity, and Simulated Colliding Object factors) on the individual $N C$ measures. The main effects of the three factors were significant: $F(1,19)=10.93, p<.001, \eta_{\mathrm{p}}{ }^{2}=.37 ; F(1,19)=30.24$, $p<.001, \eta_{\mathrm{p}}{ }^{2}=.61 ;$ and $F(5,95)=54.11, p<.001, \eta_{\mathrm{p}}{ }^{2}=.74$, respectively. The effects of the two-factor Collision Type $\times$ Precollision Velocity interaction were significant, $F(1,19)=$ $8.26, p<.01, \eta_{\mathrm{p}}{ }^{2}=.30$. The effects of the two-factor interactions for Collision Type $\times$ Simulated Colliding Object and Precollision Velocity $\times$ Simulated Colliding Object were not significant, $F(5,95)=0.96, p>.1, \eta_{\mathrm{p}}{ }^{2}=.05$, and $F(5,95)=$ $0.94, p>.1, \eta_{\mathrm{p}}{ }^{2}=.05$, respectively. Neither was the effect of the three-factor interaction significant, $F(5,95)=1.36, p>.1$, $\eta_{\mathrm{p}}^{2}=.07$.

\footnotetext{
${ }^{5}$ Figure 7 shows that there is no relationship between the size of the simulated objects and the $N C$ measures. As illustrative examples, we can see that for some pairs of simulated objects with the same size (e.g., terracotta spheres and super balls) the $N C$ s were very different from one another. Symmetrically, for some pairs of simulated objects with different sizes (e.g., tennis balls and table tennis balls), the $N C$ s were quite similar to one another. We point out, however, that the possible influence of size on the $N C$ measures would be better explored in an experiment in which the size of the colliding objects is varied independently of their simulated materials.
}

Bonferroni post-hoc comparisons (see Table 3 ) showed that the $N C$ measures for the six types of simulated colliding objects, averaged across the Collision Type and Precollision Velocity factors, were all significantly different from one another. These results support the robustness and generalizability of the main outcomes of Experiments $1 \mathrm{~A}$ and $1 \mathrm{~B}$ - that is, that participants intuitively understood that collisions between more elastic objects imply higher values of $C$ than collisions between less elastic objects.

Bonferroni-corrected post-hoc comparisons (see Table 4) also showed that the $N C$ measure, averaged across the six levels of the Simulated Colliding Object factor, was significantly lower for the head-on collision with a fast precollision velocity than for the other experimental situations. Moreover, the $N C$ measure was significantly lower for the Michottean collision with a fast precollision velocity than for the Michottean collision with a slow precollision velocity. Overall, these results indicate that the $N C$ s are also sensitive to kinematic conditions that, in real physical collisions, have no impact on restitution coefficient $C$. $^{6}$ Thus, they are examples of a discrepancy between intuitive and Newtonian

\footnotetext{
${ }^{6}$ Table 4 shows that the $N C$ measure for the Michottean collision was significantly higher than that for the head-on collision only for "fast" level of the Precollision Velocity factor. This suggests that the effects of the Collision Type factor may become evident only when the simulated collision is characterized by a relatively high precollision velocity. In Experiments $1 \mathrm{~A}$ and $1 \mathrm{~B}$, the precollision velocity was intermediate between the slow and fast levels of Experiment 2, and this may explain why, in those experiments, the effects of the Collision Type factor were somewhat unclear (i.e., its main effects were statistically significant in Exp. 1A, but not in Exp. 1B).
} 
Table 3 Results of the Bonferroni-corrected post-hoc paired comparisons for the six levels of the Simulated Colliding Object factor in Experiment 2

\begin{tabular}{|c|c|c|c|c|c|}
\hline & Terracotta & Rubber & Tennis & TableTennis & Super Ball \\
\hline Plasticine & $\begin{array}{l}t=-4.053 \\
p<.001\end{array}$ & $\begin{array}{l}t=-10.21 \\
p<.001\end{array}$ & $\begin{array}{l}t=-12.498 \\
p<.001\end{array}$ & $\begin{array}{l}t=-16.396 \\
p<.001\end{array}$ & $\begin{array}{l}t=-20.642 \\
p<.001\end{array}$ \\
\hline Terracotta & & $\begin{array}{l}t=-8.33 \\
p<.001\end{array}$ & $\begin{array}{l}t=-10.632 \\
p<.001\end{array}$ & $\begin{array}{l}t=-15.04 \\
p<.001\end{array}$ & $\begin{array}{l}t=-17.51 \\
p<.001\end{array}$ \\
\hline Rubber & & & $\begin{array}{l}t=-4.04 \\
p<.001\end{array}$ & $\begin{array}{l}t=-7.564 \\
p<.001\end{array}$ & $\begin{array}{l}t=-12.048 \\
p<.001\end{array}$ \\
\hline Tennis & & & & $\begin{array}{l}t=-3.509 \\
p<.001\end{array}$ & $\begin{array}{l}t=-6.527 \\
p<.001\end{array}$ \\
\hline Tabletennis & & & & & $\begin{array}{l}t=-3.721 \\
p<.001\end{array}$ \\
\hline
\end{tabular}

Each $t$ test had 79 degrees of freedom, and the corrected value of $\alpha$ was .0033 (i.e., .05/15)

physics of collisions, to which we shall return in the General Discussion section. We also note that, similar to what we found in Experiments 1A and 1B, the values of the NCs (averaged across the Collision Type and Precollision Velocity factors) were 0.2 to 0.5 points higher than the corresponding values of the $P C$ s.

\section{Experiment 3}

In horizontal collisions, when both spheres have the same size and material, a one-to-one relationship holds true between the kinematic pattern of a collision and the elasticity of the spheres. The relationship is specified by the following equation:

Elasticity $(A, B)=f(C)=f\left[\left(v_{B}-v_{A}\right) /\left(u_{A}-u_{B}\right)\right]$

where $f$ is a suitable increasing monotone function and the other symbols are defined as before. This equation from physics shows that, in principle, it is possible to determine the elasticity of the colliding spheres when the kinematic pattern of the collision is available.

Whereas in the previous experiments we tested the influence of objects' elasticity on the apparent "naturalness" of the kinematic patterns of collisions, in Experiment 3 we adopted a converse approach, by testing whether people can infer the elasticity of colliding objects from the kinematics of collisions. Hence, we removed the pictorial cues to the material of the spheres, varied the implicit value of parameter $C$ in the collisions, and asked the participants to rate the bounciness of the spheres' materials on the basis of the kinematics of the collisions. ${ }^{7}$ Should the participants be able to infer the elasticity of colliding objects from the kinematic pattern, then the rated bounciness of the spheres would increase as the implicit

\footnotetext{
${ }_{7}$ We asked the participants to rate "bounciness" rather than "elasticity" because the physical concept of elasticity is more akin to bounciness than to elasticity, in everyday parlance (see also note 2 ).
}

value of coefficient $C$ in the collisions increased. In Experiment 3, we also tested whether the rated bounciness was influenced by kinematic conditions that, in real collisions, are irrelevant to the value of $C$. We thus manipulated collision type and precollision velocity according to a 2 Collision Type (Michottean, head-on) $\times 2$ Precollision Velocity (fast, slow) factorial design. The implicit value of parameter $C$ in the simulated collisions was varied for each combination of levels within the two experimental factors (see the Experimental Design section below).

In a study similar to the experiment we are going to describe, Warren et al. (1987) presented their observers with virtual animations of a ball that fell from high and bounced (collided) several times upon the ground at the end of the fall. The observers were asked to rate the bounciness of the ball on the basis of the kinematic information provided in the animation. The results showed a correlation between the rated bounciness of the ball and the value of $C$ implied by the bounces. This suggests that people can "infer" the bounciness of an object on the basis of the kinematics of its bounces.

The approach we adopted in this experiment also bears some resemblance to that used in research on the visual perception of relative mass among colliding objects (Gilden \& Proffitt, 1989, 1994; Runeson, 1977/1983, 1995; Runeson \&

Table 4 Results of the Bonferroni-corrected post-hoc paired comparisons for the four combinations of Collision Type and Precollision Velocity factors in Experiment 2

\begin{tabular}{llll}
\hline & (M, Fast) & (H, Slow) & (H, Fast) \\
\hline (M, Slow) & $\boldsymbol{t}=\mathbf{2 . 8 0 3}$ & $t=2.253$ & $\boldsymbol{t}=\mathbf{8 . 0 5 6}$ \\
& $\boldsymbol{p}=\mathbf{. 0 0 6}$ & $p=.026$ & $\boldsymbol{p}<\mathbf{. 0 0 1}$ \\
(M, Fast) & & $t=0.189$ & $\boldsymbol{t}=\mathbf{6 . 3 7 2}$ \\
& & $p=.85$ & $\boldsymbol{p}<\mathbf{. 0 0 1}$ \\
(H, Slow) & & $\boldsymbol{t}=\mathbf{7 . 3 0 9}$ \\
& & & $\boldsymbol{p}<. \mathbf{0 0 1}$ \\
\hline
\end{tabular}

Each $t$ test had 119 degrees of freedom, and the corrected value of $\alpha$ was .0083 (i.e., .05/6) 
Vedeler, 1993; Todd \& Warren, 1982). For experiments in this area, observers are typically presented with simulated collisions between abstract shapes and are asked to judge the relative masses of the colliding objects. Observers are thus required to judge a stable property of objects (relative mass) on the basis of the kinematic information provided by simulated collisions.

\section{Method}

Participants Fifteen psychology students at the University of Padua (ages 20-36 years; seven females, eight males) participated in the experiment on a voluntary basis. They all had normal or corrected-to-normal visual abilities, and none of them had participated in Experiment 1A or 1B. On average, they had studied physics at school for 2.2 years $(S D=1.47$ years $)$.

Stimuli and apparatus The stimuli and apparatus were the same as in Experiments 1A and 1B, except that the simulated spheres (created by 3D Studio Max) all had a smooth, greenish appearance. The spheres had a fixed size $\left(8.4 \mathrm{~cm}^{3}\right)$; their diameters measured on the screen was $2.52 \mathrm{~cm}$, subtending a visual angle of $2.88^{\circ}$. The precollision velocity of sphere $A$ could be either 6.7 or $11.9 \mathrm{~cm} \mathrm{~s}^{-1}$ in both the Michottean and head-on collisions. The precollision velocity of $B$ was null in the Michottean collisions, whereas it could be either -6.7 or $-11.9 \mathrm{~cm} \mathrm{~s}^{-1}$ in the head-on collisions. No real spheres were presented to the participants before or during the trials.

Procedure The instructions on the screen informed the participants that they would be presented with two simulated colliding spheres and that their task was to rate, by integer numbers from 0 to 100 , the bounciness of the materials from which the colliding spheres seemed to be made. The participants were told that the materials were not visually discernible because the spheres had been covered with a very thin layer of greenish paper, and that their task was to estimate the bounciness of the hidden material on the basis of the spheres' collision behavior. They were also told that both spheres in each collision were made of the same material. The instructions further specified that the spheres could be made of different materials, such as Plasticine, iron, wood, polystyrene, rubber, and so forth, and that the participants had to respond 0 if they believed that the material was not bouncy at all (such as Plasticine). If, instead, they believed that the material was very bouncy (such as that of super balls), they had to respond 100 . Finally, if they believed that the material had an exactly intermediate bounciness between those extremes, they had to respond 50. In each trial, the participants were allowed to view the stimulus as many times as they wanted by pressing the spacebar on the keyboard; when they felt ready to respond, they had to press ENTER on the keyboard, type the chosen number, and then press ENTER again. After reading the instructions, the participants were presented with eight collisions that were representative of the entire sample of experimental stimuli, in order to familiarize them with the task.

Experimental design For each combination of levels for the factors Collision Type (Michottean, head-on) and Precollision Velocity (fast, slow), we varied the value of coefficient $C$ in six steps (values of $0,0.2,0.4,0.6,0.8,1$ ). Overall, the participants had 48 test trials, resulting from a 2 Collision Type $\times$ 2 Precollision Velocity $\times 6$ Value of $C$ Implicit in Collisions $\times$ 2 Repetition factorial design.

\section{Results and discussion}

Figure 8A shows the rated bounciness of the spheres' materials (averaged across participants and repetitions) as a function of the value of $C$ implicit in the collisions (horizontal axis) for each combination of levels for the Collision Type and Precollision Velocity factors (separate curves). Overall, the rated bounciness increased as the implicit value of $C$ in the collisions increased. The pattern of divergent curves revealed that the rate at which the judged bounciness increased (slope of the curves) was greater for head-on than for Michottean collisions and greater for the fast than for the slow precollision velocity.

In order to test this evidence statistically, we performed a three-way within-participants ANOVA (with the Value of $C$, Collision Type, and Precollision Velocity factors) on the spheres' rated bounciness values (averaged across repetitions). The main effects of the three factors were significant: $F(5,70)=$ $127.8, p<.001, \eta_{\mathrm{p}}{ }^{2}=.9 ; F(1,14)=9.94, p<.01, \eta_{\mathrm{p}}{ }^{2}=.41 ;$ and $F(1,14)=62.47, p<.001, \eta_{\mathrm{p}}{ }^{2}=.82$, respectively. The effects of the two-factor interactions for Value of $C \times$ Collision Type and Value of $C \times$ Precollision Velocity were significant, $F(5,70)=$ 22.54, $p<.001, \eta_{\mathrm{p}}{ }^{2}=.62$, and $F(5,70)=10.99, p<.001$, $\eta_{\mathrm{p}}{ }^{2}=.44$, respectively. The effects of the two-factor interaction between Collision Type and Precollision Velocity were not significant, $F(1,14)=0.51, p>.1, \eta_{\mathrm{p}}{ }^{2}=.03$, nor was the effect of the three-factor interaction, as $(5,70)=1.85, p>.1, \eta_{\mathrm{p}}{ }^{2}=.12$.

Linear regression analyses (see Fig. 8A) showed that the rate at which the judged bounciness increased as coefficient $C$ increased was greatest for the fast head-on collision (slope = 95.47 , intercept $=4.56, R^{2}=.83$ ), followed by the slow headon collision ( slope $=66.49$, intercept $=3.02, R^{2}=.69$ ), then by the fast Michottean collision (slope $=45.11$, intercept $=16.80$, $R^{2}=.29$ ), and finally by the slow Michottean collision (slope $=$ 29.53 , intercept $=11.09, R^{2}=.29$ ). As is shown by Fig. $8 \mathrm{~A}$ and these statistical results, within each of the four combined levels of the Collision Type and Precollision Velocity factors, the bounciness estimated by observers steadily increased (at different rates) as the value of coefficient $C$ increased. This general trend supports the hypothesis originating our experiment, 


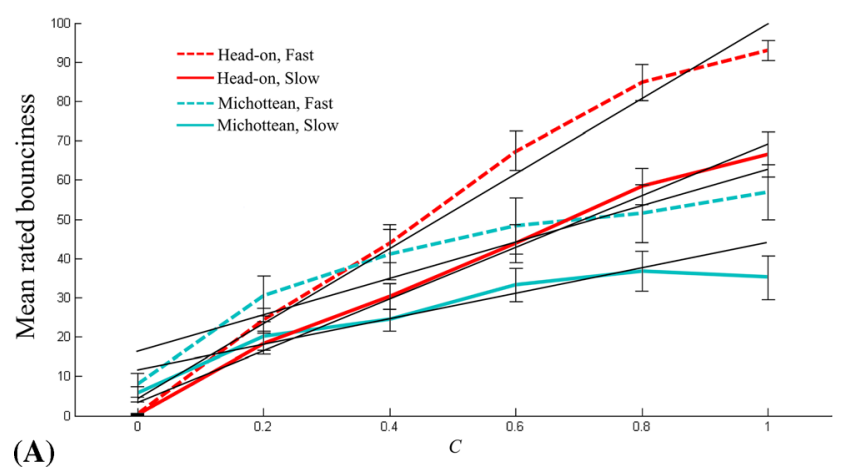

Fig. 8 (a) Mean rated bounciness from Experiment 3, represented as a function of the coefficient $C$, for each combination of the Collision Type and Precollision Velocity factors. The vertical bars represent the standard errors of the means. Regression lines (see the text) are also represented for

because it illustrates that observers can consistently infer the elasticity of spheres' materials on the basis of the kinematic patterns of collisions.

It is worth noting, however, that the participants' responses were not fully consistent with what physical science would predict. The effects of the Value of $C$ factor were conditional on the levels of the Collision Type and Precollision Velocity factors. Specifically, for any fixed value of $C$, the rated bounciness of the spheres tended to increase in passing from the Michottean to the head-on collision, and from the slow to the fast precollision velocity. Notably, both collision type and precollision velocity appear to have opposite effects on the rated bounciness in this experiment and on the $N C$ measures of Experiment 2 (see Figs. 7 and 8A for a comparison). We will provide an explanation of this apparent contrast in the General Discussion section. Below, we discuss a response model of the rated bounciness of colliding spheres that may account for the results of Experiment 3.

\section{A response model of the rated bounciness of colliding spheres}

The response model we considered is expressed by this equation:

Rated Bounciness $(A, B)=r \times\left(v_{B}-v_{A}\right)^{p} /\left(u_{A}-u_{B}\right)^{q}$,

where $u_{A}, u_{B}, v_{A}$, and $v_{B}$ are the pre- and postcollision velocities of spheres $A$ and $B$ in a collision, and $p, q$, and $r$ are the parameters to be estimated. This equation is akin to Eq. 8 above, but it is more "flexible" because it includes parameters $p$ and $q$, which may be interpreted as measures of the separate contributions of $v_{B}-v_{A}$ and $u_{A}-u_{B}$ in determining the rated bounciness of the spheres (parameter $r$ is a simple rescaling coefficient). Specifically, if $p=q$ (i.e., the relative post- and precollision velocities contribute equally to rated bounciness), then the right-hand side of Eq. 9 would amount to a special case of physical Eq. 8, in which $f(\mathrm{C})=r \times C^{p}$. In this case, the

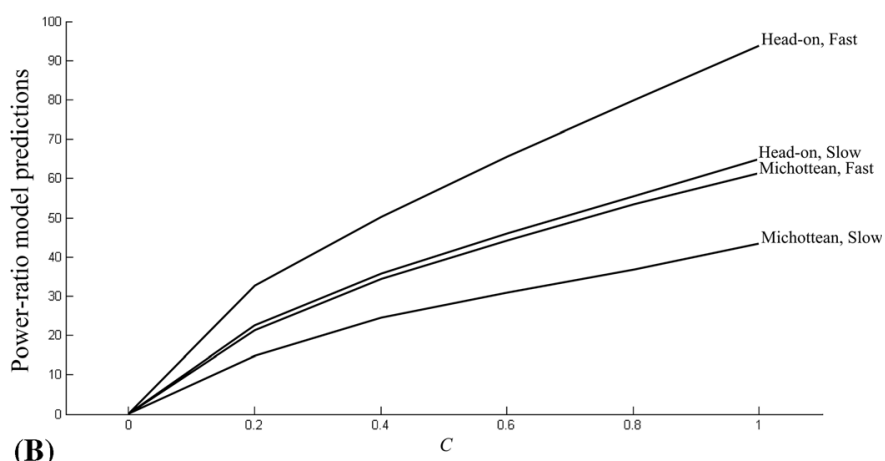

(B)

each combination of the two factors. (b) Predicted rated bounciness for each combination of the Collision Type and Precollision Velocity factors according to the power-ratio model of Eq. 9 (with $p=.669, q=0.057$, and $r=13.348)$

rated bounciness would be independent of the Collision Type and Precollision Velocity factors, and the curves corresponding to each combination of levels of the two factors would coincide. Otherwise, if $p \neq q$, the relative post- and precollision velocities would contribute unequally to the rated bounciness of the colliding spheres. This would imply a pattern of diverging curves in which the rate of variation of the rated bounciness as a function of $C$ would be dependent upon the specific combination of the levels of the Collision Type and Precollision Velocity factors. ${ }^{8}$

We fitted the response model of Eq. 9 (a power-ratio model) to the data of Experiment 3, and obtained the following estimates by the least-squares criterion: $p=.669, q=0.057$, and $r=13.348$. Figure $8 \mathrm{~b}$ shows the curves implied by Eq. 9 when these estimates are substituted for the parameters in the formula, and $u_{A}$ is set to 6.7 for slow and to 11.9 for fast precollision velocity (recall that $u_{B}=0$ in Michottean collisions and $u_{B}=-u_{A}$ in head-on collisions). We compared the performance of this model with that of the linear model that was formed of the regression lines for the four combinations of levels of the Collision Type and Precollision Velocity factors. The linear model has two parameters (intercept and slope) for each regression line (i.e., eight parameters). The statistical results for that model have been listed above and are illustrated in Fig. 8A. The square roots of the residual variance were 17.91 for the power-ratio model and 17.74 for the linear model. Thus, the power-ratio model proves almost as good as the linear model, in terms of accuracy, while being decidedly simpler with respect to the number of parameters (three rather than eight).

\footnotetext{
$\overline{{ }^{8} \text { Recall that } u_{B}}=0$ in Michottean collisions and $u_{B}=-u_{A}$ in head-on collisions; by virtue of Eq. 3, this implies that the right-hand side of Eq. 9 becomes $r \times\left(u_{A}\right)^{p-q} \times C^{p}$ for the former and $r \times\left(2 u_{A}\right)^{p-q} \times C^{p}$ for the latter type of collision. When, for instance, $p>q$, these equations imply that the rated bounciness increases more with $C$ in the case of a fast than of a slow precollision velocity. Moreover, for a fixed precollision velocity $\left(u_{A}\right)$, the rated bounciness increases more with $C$ in the case of head-on collisions than in the case of Michottean collisions [because $\left(2 u_{A}\right)^{p-q}>\left(u_{A}\right)^{p-q}$ ].
} 
The most salient feature of these results is that $p=.669$ is clearly greater than $q=0.057$. This is the algebraic reason for the kind of divergence visible in Fig. 8B (see note 8). Within the frame of the response model of Eq. 9, this means that the relative postcollision velocity proved to contribute much more to the rated bounciness of the colliding spheres than the relative precollision velocity. Significantly, similar evidence was obtained in experiments on the visual perception of the relative masses of colliding objects (Gilden \& Proffitt, 1989, 1994; Todd \& Warren, 1982). A possible interpretation of this finding is that the relative postcollision velocity was more salient to the participants than the relative precollision velocity. This may be due to the fact that, at the time of the response, the memory trace for the postcollision phase was more recent (hence, more "vivid") than that for the precollision phase. This may have prompted the participant to base his or her response more on the relative postcollision velocity than on the relative precollision velocity.

To sum up, the fact that rated bounciness increases for increasing $C$ is a sign of the consistency between intuitive and Newtonian physics. In contrast, the fact that rated bounciness depends on collision type and precollision velocity is a sign of inconsistency between intuitive and Newtonian physics, and supports the idea that the postcollision phase is more salient than the precollision phase in the task of rating the bounciness of colliding spheres.

\section{General discussion}

From Paolo Bozzi's (1959) seminal work onward, people's intuitive understanding of the relationship between force and motion has been explored using simulations of physical events involving abstract, "immaterial" objects. We undertook our present study in light of the belief that a deeper understanding of the force-motion topic requires the use of more realistic physical simulations involving "material" stimuli.

On the whole, the results that we obtained support the hypothesis that people intuitively understand the relationship between the elasticity of objects and the kinematic patterns of collisions. Specifically, the results of Experiments 1A, 1B, and 2 showed that the implicit coefficient $C$ in collisions that appeared "natural" consistently varied with the simulated materials of the colliding spheres. Moreover, the results of Experiment 3 showed that the rated bounciness of the colliding spheres consistently depended on the implicit coefficient $C$ in the collisions. These findings are in line with the results of recent studies (Lupo \& Barnett-Cowan, 2015; Vicovaro \& Burigana, 2014) showing that people properly take account of material properties when asked to judge the physical behavior of objects.
On the influences of collision type and precollision velocity on the judged naturalness of collisions

The results of our experiments also highlighted some discrepancies between intuitive and Newtonian physics of collisions. One discrepancy is the influence (i.e., the main and interaction effects) of collision type and precollision velocity both on the participants' judgments of the naturalness of collisions (Exp. 2) and on their judgments of the bounciness of colliding spheres (Exp. 3). Above we provided a tentative explanation of the results of Experiment 3, in terms of a greater saliency of the postcollision phase on participants' bounciness ratings. The explanation of the results of Experiment 2 that we are now going to suggest is closely related to that conjecture.

Let us presume that when both the simulated material of the colliding objects and the kinematic pattern of the collision are available, which is the case in Experiment 2, the participant infers the elasticity of the objects from both of these sources of stimulus information. We call the "elasticity from the material cues" that implied by the simulated material, and the "elasticity from the kinematic cues" that implied by the kinematics of the collision. ${ }^{9}$ Let us also presume that a simulated collision is judged as "natural" or "unnatural" depending on the consistency or inconsistency between the elasticity from the material cues and the elasticity from the kinematic cues. For instance, in a head-on collision between Plasticine spheres with $C=\left(v_{B}-v_{A}\right) /\left(u_{A}-u_{B}\right)=1$, presumably the elasticity suggested by the material would appear much lower than that suggested by the kinematics, which would prompt an "unnatural" response. These assumptions caused us to conjecture that, in Experiment 2, the participants adjusted the parameter $C$ implicit in collisions until the elasticity from the kinematics matched the elasticity from the material.

Now, in Experiment 3, only the kinematic cues to elasticity were in action and we saw that, for any fixed value of $C$, the four experimental conditions formed the sequence (Michottean slow, Michottean fast, head-on slow, head-on fast) when ordered by the increasing rated bounciness (see Fig. 8). We interpreted this result as being attributed to the saliency of the relative postcollision velocity. By contrast, in Experiment 2, the material cues and the kinematic cues were both in action and, following the interpretation in the preceding paragraph, the participants' task was to adjust parameter $C$ so that a match between elasticity from the material (fixed) and elasticity from the kinematics was attained. In these conditions, and according to a basic compensation principle (if $a$ $+b=c+d$ and $a<c$, then $b>d$ ), it is only natural to expect that, for a fixed material, the order between the four

\footnotetext{
${ }^{9}$ At the current stage of our research, we cannot draw any conclusion about the psychological processes related to the participants' judgments of "elasticity from material cues." We can only speculate that both visual and haptic perceptual processes might be involved, as well as high-level cognitive processes related to the participant's past experiences.
} 


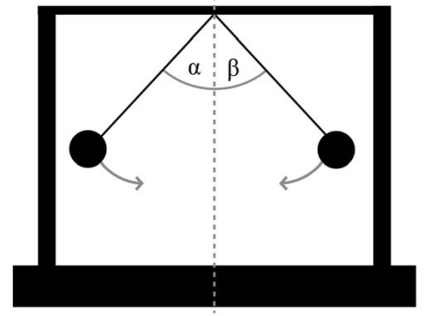

1. Pre-collision phase

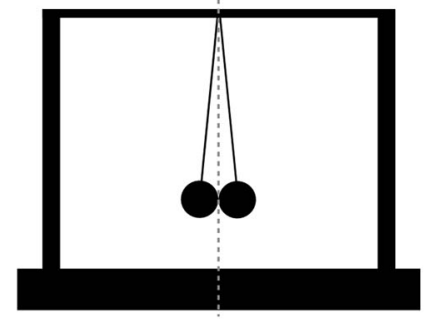

2. Collision phase

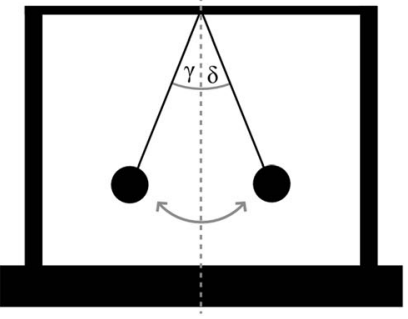

3. Post-collision phase

Fig. 9 Three frames of a collision between two pendulum spheres. The symbols $\alpha, \beta, \gamma$, and $\delta$ have been added for reference in the text. Arrows have been added to indicate which objects are moving at the three stages of the collision event

experimental conditions found by increasing the adjusted value of $C$ would be the opposite of the order mentioned above (i.e., it would be head-on fast, head-on slow, Michottean fast, Michottean slow). Actually, with few exceptions, this was the order revealed by the data of Experiment 2 (see Fig. 7).

To sum up, if our assumptions about the psychological processes underlying the experimental tasks are correct, then the (physically unexpected) influences of the collision type and precollision velocity on the results of Experiments 2 and 3 allow for a unified explanation. This relies on the hypothesis that the elasticity inferable from the kinematic pattern of a collision depends more strongly on the postcollision than on the precollision phase of the event.

\section{On the violation of the principle of energy conservation}

The results of our experiments also highlight another noteworthy discrepancy between the intuitive and Newtonian physics of collisions. Observers tend to overestimate the implicit value of $C$ in "natural" collisions and judge simulated collisions that are formally inconsistent with the principle of energy conservation $(C>1)$ as "natural." This evidence does not fit the assumption that people may have "internalized" a physically plausible value of $C$ "in order to reflect knowledge on the natural statistics of our ecological environment" (De Sá Teixeira et al., 2014, p. 499). nor the assumption that observers are highly sensitive to violations of the principle of energy conservation (Twardy \& Bingham, 2002). In particular, the discrepancies between our results and those of Twardy and Bingham are worthy of comment.

Twardy and Bingham (2002) presented their participants with virtual animations depicting a ball falling from high up that bounced (collided) several times upon the ground at the end of the fall. They manipulated the value of $C$ implied by the rebounds of the ball and asked the participants to rate the "naturalness" of the motion. The results showed that the naturalness ratings sharply decreased when the simulated value of $C$ was greater than $1-$ namely, when the animation was inconsistent with the principle of energy conservation. At least three sources of information are relevant in the case of a ball bouncing several times upon the ground: the relative height of the bounces, their relative period, and the relative bounce velocity. These sources are abundant because they repeat themselves in the sequence of bounces. On the contrary, in the case of horizontal collisions (our stimuli), only the ratio between the relative pre- and postcollision velocities of the spheres (right part of Eq. 3) provides information about possible violations of the principle of energy conservation. A reason why people tend to judge simulated bounces implying $C>1$ as "unnatural" but simulated horizontal collisions as "natural" in the same condition might be the greater amount of stimulus information available in the former situation. This highlights that the outcomes of intuitive physics studies should be interpreted with caution. Our picture of people's intuitive understanding of the laws of physics appears to depend on the specific features of the stimuli used in empirical research (cf. Cooke \& Breedin, 1994, on the "context specificity" of intuitive physics). ${ }^{10}$

Author note We thank Veronica Quaini for help in collecting the data of Experiments $1 \mathrm{~A}$ and $1 \mathrm{~B}$.

\section{Appendix}

The measures of $P C$ (physical coefficient of restitution) for the experimental conditions of Experiments 1A, 1B, and 2 were obtained using a pendulum device, as is illustrated in Fig. 9. The pendulum spheres are the real spheres referred to in the text. We used a pendulum because it obeys physical principles similar to those involved in horizontal collisions; in particular, the spin of the spheres and the resistance of the medium are

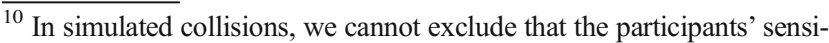
tivity to violations of the principle of kinetic energy might increase with the realism of the motion of the colliding objects. In this regard, in future studies it might be worthwhile to explore the effects of adding rotation to the colliding objects, and/or the effects of making the colliding objects move in response to the application of an external force (e.g., the hit of a billiard cue).
} 
(ideally) negligible. In the case of pendulum collisions, $C$ can be determined by this equation:

$C=[[\sqrt{ }(1-\cos \delta)-\sqrt{ }(1-\cos \gamma)] /[\sqrt{ }(1-\cos \alpha))-\sqrt{ }(1-\cos \beta)]$,

where $\alpha, \beta, \gamma$, and $\delta$ denote the angles (in radians) represented in Fig. 9 (for Michottean collisions, $\beta=0$ ).

For each experimental condition, measures of $P C$ were obtained by causing collisions between the pendulum spheres and measuring the angles required by the righthand part of Eq. 10. For instance, in the case of head-on collisions, we first raised the spheres by hand to certain angles $\alpha$ and $\beta$. Then we released the spheres simultaneously and measured the resulting postcollision angles $\gamma$ and $\delta$. We used the same procedure for the Michottean collisions, except that only one sphere was raised before the collision. Accurate measures of the pre- and postcollision angles were obtained from videotapes of each collision. We recorded several collisions for each experimental condition while randomly varying the precollision angles $\alpha$ and $\beta$. Among the recorded collisions, we chose ten "valid" collisions that had the least lateral and/or rotational sphere motion. We computed $C$ for each of the ten collisions using Eq. 10, and the $P C$ s were obtained by averaging the ten values of $C$ for each experimental condition.

\section{References}

Anderson, N. H. (1981). Foundations of information integration theory. New York: Academic Press.

Anderson, N. H. (1983). Intuitive physics: Understanding and learning of physical relations. In T. J. Tighe \& B. E. Shepp (Eds.), Perception, cognition, and development: Interactional analysis (pp. 231-265). Hillsdale: Erlbaum.

Bae, G. Y., \& Flombaum, J. I. (2011). Amodal causal capture in the tunnel effect. Perception, 40, 74-90.

Barnes, G. (1958). Study of collisions.Part I. A survey of the periodical literature. American Journal of Physics, 26, 5-8. doi:10.1119/1. 1934583

Bozzi, P. (1959). Le condizioni del movimento "naturale" lungo i piani inclinati. Rivista di Psicologia, 53, 337-352.

Cooke, N. J., \& Breedin, S. D. (1994). Constructing naive theories of motion on the fly. Memory \& Cognition, 22, 474-493. doi:10.3758/ BF03200871

Cross, R. (1999). The bounce of a ball. American Journal of Physics, 67, 222-227. doi:10.1119/1.19229

De Sá Teixeira, N. A., Oliveira, A. M., \& Duarte Silva, A. (2014). An information integration study on the intuitive physics of the Newton's cradle. Psicológica, 35, 479-502.

Gilden, D. L., \& Proffitt, D. R. (1989). Understanding collision dynamics. Journal of Experimental Psychology: Human Perception and Performance, 15, 372-383. doi:10.1037/00961523.15.2.372

Gilden, D. L., \& Proffitt, D. R. (1994). Heuristic judgment of mass ratio in two-body collisions. Perception \& Psychophysics, 56, 708-720. doi:10.3758/BF03208364
Hecht, H. (2001). Regularities of the physical world and the absence of their internalization. Behavioral and Brain Sciences, 24, 608-617. doi:10.1017/S0140525X01000036

Hecht, H., \& Bertamini, M. (2000). Understanding projectile acceleration. Journal of Experimental Psychology: Human Perception and Performance, 26, 730-746. doi:10.1037/0096-1523.26.2.730

Kaiser, M. K., \& Proffitt, D. R. (1987). Observers' sensitivity to dynamic anomalies in collisions. Perception \& Psychophysics, 42, 275-280. doi:10.3758/BF03203079

Kaiser, M. K., Proffitt, D. R., \& Anderson, K. (1985a). Judgments of natural and anomalous trajectories in the presence and absence of motion. Journal of Experimental Psychology: Learning, Memory, and Cognition, 11, 795-803. doi:10.1037/0278-7393.11.1-4.795

Kaiser, M. K., Proffitt, D. R., \& McCloskey, M. (1985b). The development of beliefs about falling objects. Perception \& Psychophysics, 38, 533-539. doi:10.3758/BF03207062

Kaiser, M. K., Proffitt, D. R., Whelan, S. M., \& Hecht, H. (1992). Influence of animation on dynamical judgments. Journal of Experimental Psychology: Human Perception and Performance, 18, 669-689. doi:10.1037/0096-1523.18.3.669

Kingdom, F. A. A., \& Prins, N. (2010). Psychophysics: A practical introduction. San Diego: Academic Press.

Kittel, C., Knight, W. D., \& Ruderman, M. A. (1973). Mechanics. New York: McGraw-Hill.

Krist, H. (2000). Development of naive beliefs about moving objects: The straight-down belief in action. Cognitive Development, 15, 281308. doi:10.1016/S0885-2014(00)00029-0

Levitt, H. (1971). Transformed up-down methods in psychoacoustics. Journal of the Acoustical Society of America, 49, 467-477. doi:10. $1121 / 1.1912375$

Lupo, J., \& Barnett-Cowan, M. (2015). Perceived object stability depends on shape and material properties. Vision Research, 109, 158-165. doi:10.1016/j.visres.2014.11.004

McCloskey, M. (1983). Intuitive physics. Scientific American, 248, 122130.

McCloskey, M., Caramazza, A., \& Green, B. (1980). Curvilinear motion in the absence of external forces: Naïve beliefs about the motion of objects. Science, 210, 1139-1141. doi:10.1126/science.210.4474. 1139

McCloskey, M., \& Kohl, D. (1983). Naive physics: The curvilinear impetus principle and its role in interactions with moving objects. Journal of Experimental Psychology: Learning, Memory, and Cognition, 9, 146-156. doi:10.1037/02787393.9.1.146

McCloskey, M., Washburn, A., \& Felch, L. (1983). Intuitive physics: The straight-down belief and its origin. Journal of Experimental Psychology: Learning, Memory, and Cognition, 9, 636-649. doi: 10.1037/0278-7393.9.4.636

Michotte, A. (1963). The perception of causality. London: Methuen.

O'Sullivan, C., Dingliana, J., Giang, T., \& Kaiser, M. K. (2003). Evaluating the visual fidelity of physically based animations. ACM Transactions on Graphics, 22, 527-536. doi:10.1145/882262. 882303

Proffitt, D. R., \& Gilden, D. L. (1989). Understanding natural dynamics. Journal of Experimental Psychology: Human Perception and Performance, 15, 384-393. doi:10.1037/0096-1523.15.2.384

Runeson, S. (1983). On visual perception of dynamic events (Acta Universitatis Upsaliensis: Studia Psychologica Upsaliensia, Serial No. 9). Stockholm: Almqvist \& Wiksell (Original work published 1977).

Runeson, S. (1995). Support for the cue-heuristic model is based on suboptimal observer performance: Response to Gilden and Proffitt (1994). Perception \& Psychophysics, 57, 1262-1273. doi:10.3758/ BF03208381

Runeson, S., \& Frykholm, G. (1983). Kinematic specification of dynamics as an informational basis for person-and-action 
perception: Expectation, gender recognition, and deceptive intention. Journal of Experimental Psychology: General, 112, 585-615. doi:10.1037/0096-3445.112.4.585

Runeson, S., \& Vedeler, D. (1993). The indispensability of precollision kinematics in the visual perception of relative mass. Perception \& Psychophysics, 53, 617-632. doi:10.3758/BF03211738

Sanborn, A. N., Mansinghka, V. K., \& Griffiths, T. L. (2013). Reconciling intuitive physics and Newtonian mechanics for colliding objects. Psychological Review, 120, 411-437. doi:10.1037/a0031912

Scholl, B. J., \& Nakayama, K. (2002). Causal capture: Contextual effects on the perception of collision events. Psychological Science, 13, 493-498. doi:10.1111/1467-9280.00487

Todd, J. T., \& Warren, W. H. (1982). Visual perception of relative mass in dynamic events. Perception, 11, 325-335. doi:10. 1068/p110325

Twardy, C. R., \& Bingham, G. P. (2002). Causation, causal perception, and conservation laws. Perception \& Psychophysics, 64, 956-968. doi:10.3758/BF03196799
Vicovaro, M., \& Burigana, L. (2014). Intuitive understanding of the relation between velocities and masses in simulated collisions. Visual Cognition, 22, 896-919. doi:10.1080/13506285.2014.933940

Vicovaro, M., Hoyet, L., Burigana, L., \& O’Sullivan, C. (2014). Perceptual evaluation of motion editing for realistic throwing animations. $A C M$ Transactions on Applied Perception, 11, 10. doi:10.1145/2617916

Warren, W. H., Kim, E. E., \& Husney, R. (1987). The way the ball bounces: Visual and auditory perception of elasticity and control of the bounce pass. Perception, 16, 309-336. doi:10.1068/p160309

White, P. A. (2007). Impressions of force in visual perception of collision events: A test of the causal asymmetry hypothesis. Psychonomic Bulletin \& Review, 14, 647-652. doi:10.3758/BF03196815

Yates, J., Bessman, M., Dunne, M., Jertson, D., Sly, K., \& Wendelboe, B. (1988). Are conceptions of motion based on a naive theory or on prototypes? Cognition, 29, 251-275. doi:10.1016/0010-0277(88)90026-1

Yela, M. (1952). Phenomenal causation at a distance. Quarterly Journal of Experimental Psychology, 4, 139-154. doi:10. 1080/17470215208416612 